\title{
Sol-gel Synthesis of Metal-Phenolic Coordination Spheres and Their Derived Carbon Composites
}

\author{
Jing Wei, ${ }^{[a]}$ Gen Wang, ${ }^{[a]}$ Feng Chen, ${ }^{[a]}$ Min Bai, ${ }^{[a]}$ Yan Liang, ${ }^{[b]}$ Huanting Wang,${ }^{[b]}$ Dongyuan Zhao, ${ }^{[c]}$ \\ Yongxi Zhao ${ }^{[a] *}$
}

\begin{abstract}
Metal-organic coordination spheres integrate the advantages of coordination polymers with spherical colloids, which are crucial for the fabrication of functional composites. However, it still lacks a universal strategy to develop chemically tailorable colloidal spheres from metal-organic coordination polymers. Here we report a formaldehyde-assisted metal-ligand crosslinking strategy for the synthesis of metal-phenolic coordination spheres based on the principle of sol-gel chemistry. A range of mono-metal (Co, $\mathrm{Fe}, \mathrm{Al}, \mathrm{Ni}$, $\mathrm{Cu}, \mathrm{Zn}$ and $\mathrm{Ce}$ ), bi-metal (Fe-Co, Co-Zn) and multi-metal (Fe-Co-Ni$\mathrm{Cu}-\mathrm{Zn})$ species can be successfully incorporated into the frameworks of the colloidal spheres. The formation of coordination spheres involves the pre-crosslinking of plant polyphenol (i.e. tannic acid) by formaldehyde in alkaline ethanol/water solvents, followed by the aggregation assembly of polyphenol oligomers via metal-ligand crosslinking. The coordination spheres can be used as sensing platforms for the analysis of nucleic acid variants with singlenucleotide discrimination, and a versatile precursor for electrode materials with high electrocatalytic performance.
\end{abstract}

Colloidal spheres have been widely applied in optics, biomedicine, biosensor, catalysis, and energy. ${ }^{[1]}$ Various methods, such as sol-gel process and hydrothermal synthesis, have been developed to prepare colloidal spheres. As a typical case, Stöber method has been widely used to synthesize colloidal silica spheres in the alkaline alcohol/water solvents. ${ }^{\left[{ }^{[2]}\right.}$ Based on the sol-gel synthesis, a variety of inorganic and polymeric spheres (such as resorcinol-formaldehyde resin spheres) were obtained. ${ }^{[3]}$ Most importantly, combining with other techniques (e.g. spin coating), various functional composites could be fabricated, which would greatly extend the applications of colloidal spheres. ${ }^{[4]}$ Metal-organic coordination polymers have attracted intensive attentions due to their unique structure and morphology, tailorable compositions, as well as numerous applications. ${ }^{[5]}$ The integration of the advantages of

[a] Prof. J. Wei, Dr. G. Wang, Dr. F. Chen, M. Bai, Prof. Y. Zhao School of Life Science and Technology, The Key Laboratory of Biomedical Information Engineering of Ministry of Education, Xi'an Jiaotong University

Xi'an, Shaanxi 710049, P. R. China

E-mail: yxzhao@mail.xitu.edu.cn

[b] Dr. Y. Liang, Prof. H. Wang,

Department of Chemical Engineering, Monash University Clayton, Victoria 3800, Australia

[c] Prof. D. Zhao,

Department of Chemistry, State Key Laboratory of Molecula Engineering of Polymers, Shanghai Key Lab of Molecular Catalysis and Innovative Materials, iChEM, Fudan University, Shanghai 200433, P. R. China

Supporting information for this article is given via a link at the end of the document. coordination polymers with spherical structure would endow them more unique features and extend their applications. ${ }^{[6]}$

Plant polyphenols (e.g. tannic acid, TA) contain large amount of catechol or galloyl groups, which have strong chelate ability with various metal ions. The metal-TA coordination polymers, as a subclass of metal-organic coordination polymers, have recently been investigated as a versatile platform for the functional surface engineering. ${ }^{[7]}$ As the polyphenols are known to strongly bind to various substrates throng covalent and noncovalent, ${ }^{[8]}$ the controlling over the morphology and structure of such metal-phenolic coordination polymers usually relies on the shape of the external substrate (or template). ${ }^{\text {[7a] }}$ Dopamine molecule contains one catechol group, which can chelate with metal ions. Metal-loaded polydopamine spheres with tunable compositions have been well studied. ${ }^{[9]}$ However, there is very few examples for the synthesis of metal-plant polyphenol coordination spheres. Each TA $\left(\mathrm{C}_{76} \mathrm{H}_{52} \mathrm{O}_{46}\right)$ molecule contains five catechol groups and five galloyl groups to chelate with metal ions (Figure 1a). The self-assembly between TA and metal ions is more complicated than that of dopamine. Till now, most of the metal-phenolic coordination spheres were synthesized using the inorganic (or organic) sphere as a sacrificed template, resulting into a hollow structure. Ejima et al successfully synthesized metal-TA coordination polymer capsules via a layer-by-layer assembly strategy using polystyrene spheres as a sacrificed template. ${ }^{[7 a]}$ Due to the complicated polymerization process of the TA molecule and strong coordination interactions between TA and metal ions, the synthesis of metal-TA coordination spheres without using any spherical template is still a huge challenge. ${ }^{[0]}$

Herein, inspired from Stöber method for the synthesis of colloidal spheres, we report a formaldehyde-assisted metalligand crosslinking strategy for the synthesis of metal-phenolic coordination spheres with uniform diameter ( $300 \mathrm{~nm})$ and tunable compositions. The formation of spherical metal-phenolic coordination polymer involves the pre-crosslinking of TA by formaldehyde in the alkaline ethanol/water solvents, followed by the aggregation assembly of polyphenol oligomers via metalligand crosslinking. Due to the diverse chelation ability of TA, a range of mono-metal (such as $\mathrm{Co}^{2+}, \mathrm{Fe}^{2+}, \mathrm{Fe}^{3+}, \mathrm{Al}^{3+}, \mathrm{Ni}^{2+}, \mathrm{Cu}^{2+}$, $\mathrm{Zn}^{2+}$ and $\mathrm{Ce}^{3+}$ ), bi-metal (such as Fe-Co, Co- Zn), and multimetal (such as Fe-Co-Ni-Cu-Zn) species can be successfully incorporated into the frameworks of the coordination spheres. Due to the unique sperical structure and metal-phenolic network, these coordination spheres can be applied for reliable analysis of nucleic acid variants with single-nucleotide discrimination. In addition, such coordonation sphere derived nanoporous $\mathrm{M} / \mathrm{N}-\mathrm{C}$ ( $\mathrm{M}=\mathrm{Fe}$, Co) composites exhibit high onset potential, large limiting current density and good stability for oxygen reduction reaction (ORR). 

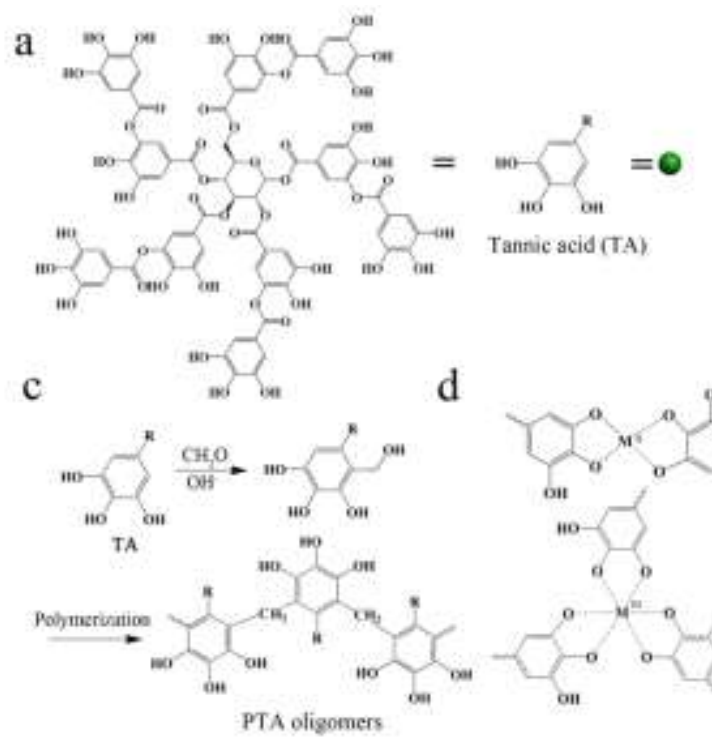

b
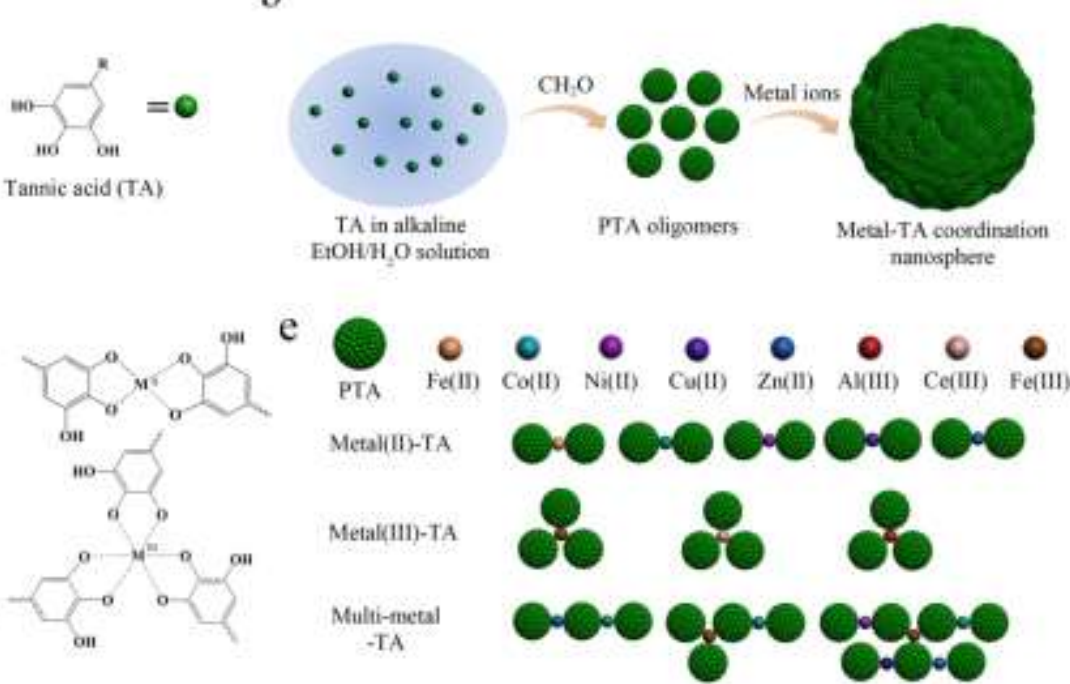

Figure 1 (a) Chemical structure of TA. (b) The synthesis of metal-phenolic coordination spheres. Step 1, the polymerization of TA by formaldehyde to form poly(tannic acid) oligomers; Step 2, the assembly of the oligomers to metal-TA coordination spheres via metal-ligand crosslinking. (c) The possible formation of PTA oligomers via formaldehyde crosslinking. (d) The formation of metal-phenolic bond. (e) The assembly units of metal-TA with various metal ions.

The synthesis of metal-phenolic coordination spheres mainly includes two steps (Figure 1b). The first step is similar to the extended "Stöber" method for the synthesis of resorcinolformaldehyde resin spheres. ${ }^{[3 \mathrm{~d}]}$ TA molecules were crosslinked by formaldehyde in the alkaline ethanol/water solvents (Figure 1c, Figure S1). As Pluronic F127 (polyethylene oxide-blockpolypropylene oxide-block-polyethylene oxide) can interact with TA via hydrogen bonding, TA molecules may be trapped in the matrix of F127 block copolymers. As a result, TA molecules can be polymerized moderately by formaldehyde to form poly(tannic acid) (PTA) oligomers with the assistance of F127. In the second step, the polymer oligomers were further aggregated together to form metal-TA coordination spheres due to the strong metalligand coordination interactions between the metal ions (such as $\mathrm{Co}^{2+}$ ) and catechol groups from polymer oligomers (Figure 1d). As the catechol groups have strong chelate ability with various metal ions, mono-metal $\mathrm{CO}^{2+}, \mathrm{Fe}^{2+}, \mathrm{Fe}^{3+}, \mathrm{Al}^{3+}, \mathrm{Ni}^{2+}, \mathrm{Cu}^{2+}, \mathrm{Zn}^{2+}$ and $\mathrm{Ce}^{3+}$ ), bi-metal (such as $\mathrm{Zn}-\mathrm{Co}$ and $\mathrm{Fe}-\mathrm{Co}$ ) and multi-metal species (Fe-Co-Ni-Cu-Zn) could be used as a crosslinker (Figure 1e). In order to make the coordination spheres more stable, hydrothermal treatment was further applied to crosslink the organic ligands. Moreover, the TA polymer spheres without any metal species were also synthesized by direct hydrothermal treatment of PTA oligomers.

Scanning electron microscopy (SEM) images for PTA without using any metal crosslinker show uniform spherical morphology, with diameter of around $300 \mathrm{~nm}$ (Figure 2a). When metal ions are used as a crosslinker, the obtained polymers also exhibit spherical morphology (Figure 2b, c, Figure S2a-f), indicating the formation of metal-phenolic coordination spheres. In this work, eight kinds of metal ions such as $\mathrm{Co}^{2+}, \mathrm{Fe}^{2+}, \mathrm{Fe}^{3+}, \mathrm{Al}^{3+}, \mathrm{Ni}^{2+}, \mathrm{Cu}^{2+}$ $\mathrm{Zn}^{2+}$ and $\mathrm{Ce}^{3+}$ are used as a crosslinker. All the obtained metalphenolic coordination polymers show spherical morphology. To further validate the universality of this strategy, mixed metal precursors such as $\mathrm{Co}\left(\mathrm{NO}_{3}\right)_{2} / \mathrm{Fe}\left(\mathrm{NO}_{3}\right)_{3}, \mathrm{Co}\left(\mathrm{NO}_{3}\right)_{2} / \mathrm{Zn}\left(\mathrm{NO}_{3}\right)_{2}$,
$\mathrm{Co}\left(\mathrm{NO}_{3}\right)_{2} / \mathrm{FeSO}_{4}$ and $\mathrm{Fe}\left(\mathrm{NO}_{3}\right)_{3} / \mathrm{Co}\left(\mathrm{NO}_{3}\right)_{2} / \mathrm{Ni}\left(\mathrm{NO}_{3}\right)_{2} / \mathrm{Cu}\left(\mathrm{NO}_{3}\right)_{2} / \mathrm{Zn}\left(\mathrm{NO}_{3}\right)_{2}$ were used as a metal source for crosslinking. Consequently, bimetal-TA (Co(II)$\mathrm{Fe}(\mathrm{III})-\mathrm{TA}, \mathrm{Co}(\mathrm{II})-\mathrm{Zn}(\mathrm{II})-\mathrm{TA}$ and $\mathrm{Co}(\mathrm{II})-\mathrm{Fe}(\mathrm{II})-\mathrm{TA}$ ) and multi-metalTA (Fe(III)-Co(II)-Ni(II)-Cu(II)-Zn(II)-TA) coordination spheres were obtained respectively. All these metal-phenolic coordination polymers reveal the spherical morphology and uniform diameter (Figure 2d, e, f, Figure S2g). The element mapping results for $\mathrm{Co}(\mathrm{II})-\mathrm{Fe}(\mathrm{III})-\mathrm{TA}$ reveal that $\mathrm{Co}$ and $\mathrm{Fe}$ species are distributed uniformly in the coordination spheres (Figure S3). Even for the $\mathrm{Fe}(\mathrm{III})-\mathrm{Co}(\mathrm{II})-\mathrm{Ni}(\mathrm{II})-\mathrm{Cu}(\mathrm{II})-\mathrm{Zn}(\mathrm{II})-\mathrm{TA}$ coordination polymer sphere, it can be clearly seen that $\mathrm{Fe}$, Co, $\mathrm{Ni}, \mathrm{Cu}$ and $\mathrm{Zn}$ elements are dispersed in the polymer spheres uniformly (Figure 2g).

The metal contents for the metal-phenolic coordination polymers can also be easily adjusted by changing the amount of metal source. When the mass ratio of cobalt source (i.e. $\left.\mathrm{Co}\left(\mathrm{NO}_{3}\right)_{2} \cdot 6 \mathrm{H}_{2} \mathrm{O}\right)$ to TA increased from 5 to $80 \mathrm{wt} \%$, all the obtained Co-TA coordination polymers show spherical morphology (Figure S3a-d). The content of cobalt in the coordination polymers increases from 2.3 to $12.6 \mathrm{wt} \%$ calculating from thermogravimetric analysis (Figure S4e, Figure 2h). Moreover, the compositions of bimetal-TA coordination sphere can also be simply adjusted by changing the ratio of metal sources. For example, when the molar ratio of $\mathrm{Co}$ (II) to $\mathrm{Fe}$ (III) precursor is $0.8: 1,1.4: 1$ and $2.8: 1$ respectively, the obtained $\mathrm{Co}$ (II)-Fe(III)-TA coordination polymers show spherical morphology (Figure S5). Energy dispersive X-ray analysis (EDX) results reveal the molar ratio of $\mathrm{Co}(\mathrm{II})$ to $\mathrm{Fe}(\mathrm{III})$ is $0.6: 1,1.2: 1$ and $2.5: 1$ respectively (Figure $2 \mathrm{i}$ ).

The X-ray diffraction (XRD) results prove that the metalphenolic coordination spheres show amorphous structure (Figure S4f). $\mathrm{N}_{2}$ sorption isotherms reveal that these coordination spheres show very low surface area (10-20 $\left.\mathrm{m}^{2} \mathrm{~g}^{-1}\right)$, indicating nearly no nanopores in the polymer framework (Figure 
S6, Table S1). X-ray photoelectron spectroscopy (XPS) of CoTA coordination sphere further demonstrates the existence of cobalt species in the polymer (Figure S7a). The high-resolution Co $2 p$ spectrum reveals two peaks at 779.96 and $783.79 \mathrm{eV}$, which can be ascribed to the $\mathrm{Co}$ (II)-O and $\mathrm{Co}$ (II) salts respectively (Figure S7b). ${ }^{[11]}$ The formation of $\mathrm{Co}(\mathrm{II})-\mathrm{O}$ bonds in the Co-TA coordination spheres can be further confirmed by the Raman spectra (Figure S8).
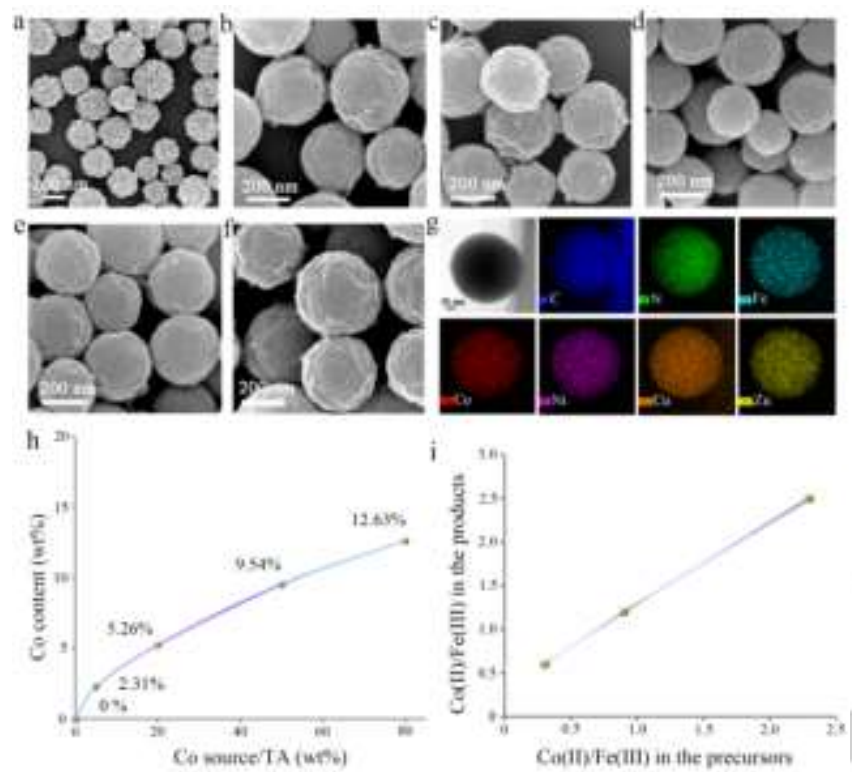

Figure 2 SEM images of (a) PTA spheres, (b) Co(II)-TA, (c) Fe(III)-TA, (d) $\mathrm{Co}$ (II)-Zn(II)-TA, (e) Co(II)-Fe(III)-TA and (f) Fe(III)-Co(II)-Ni(II)-Cu(II)-Zn(II)-TA coordination polymer spheres. (g) TEM and corresponding element mapping images for $\mathrm{Fe}(\mathrm{III})-\mathrm{Co}$ (II)-Ni(II)-Cu(II)-Zn(II)-TA coordination polymer spheres. (h) The cobalt contents in the Co-TA spheres when the mass ratio of cobalt source to TA increases. (i) The molar ratio of $\mathrm{Co}$ (II) to $\mathrm{Fe}$ (III) in the $\mathrm{Co}$ (II)$\mathrm{Fe}$ (III) coordination spheres when the molar ratio of $\mathrm{Co}$ (II) to $\mathrm{Fe}$ (III) in the precursors increases.

In order to investigate the possible formation mechanism of the metal-phenolic coordination spheres, the samples at different stages were characterized by SEM. In the first step, the TA molecules were crosslinked by formaldehyde for 24 hours. SEM results show the colloidal spheres are not formed yet at this stage (Figure 3a). When the metal source (i.e. cobalt nitrate) was added into the TA-formaldehyde solution, the metal-TA coordination spheres were formed after stirring for $5 \mathrm{~min}$ (Figure $3 b)$. After further stirring for 24 hours, the spherical structure was not changed obviously (Figure 3c). After hydrothermal treatment at $100 \stackrel{\circ}{\circ}$ for 24 hours, the spherical morphology retains. The metal-TA coordination spheres before hydrothermal treatment can be quickly dissolved in $0.1 \mathrm{M} \mathrm{HCl}$ solution. This indicates the polymer spheres were formed via metal-ligand crosslinking (Figure 3e, f, g). Comparably, the metal-TA coordination spheres after hydrothermal treatment is stable in $0.1 \mathrm{M} \mathrm{HCl}$ at least for 12 hours (Figure $3 \mathrm{~h}$ ). These results prove the organic ligands in the coordination spheres can be further polymerized during hydrothermal treatment, which can stabilize the spherical morphology and dramatically improve the acidic tolerance of the coordination spheres (Figure 3i). When the formaldehyde was absent in the synthesis, only metal-TA rods were obtained (Figure $3 \mathrm{~d}$ ), indicating an important role of formaldehyde in the formation of coordination sphere.
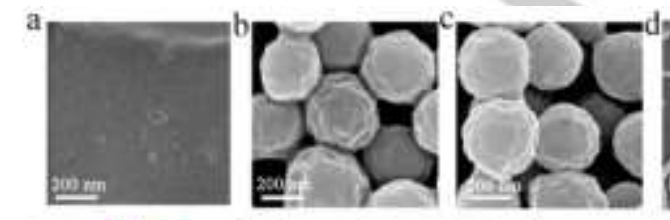

e
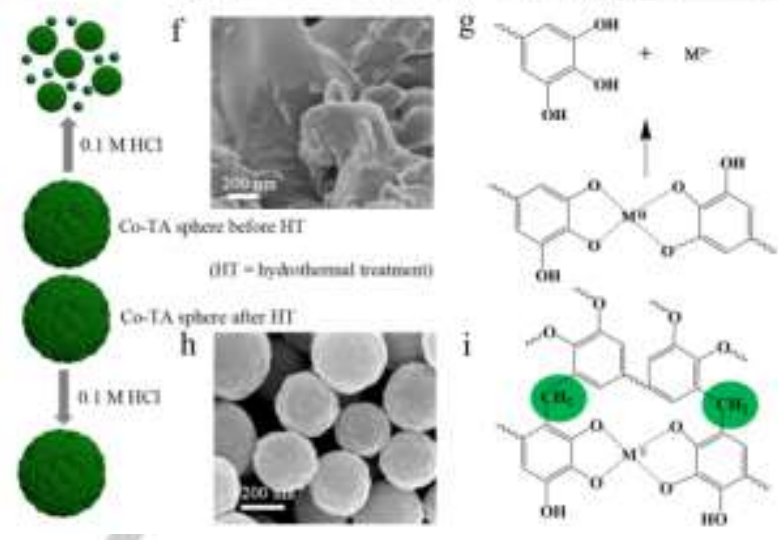

Figure 3 SEM images of (a) PTA oligomers before addition of metal ions, CoTA coordination spheres after addition of cobalt ions for (b) $5 \mathrm{~min}$, (c) 24 hours, and (d) Co-TA coordination rods without using formaldehyde as a crosslinker. (e) Schematic for the stability of the Co-TA coordination in the acid conditions. SEM images of ( $f$ ) Co-TA spheres without hydrothermal treatment $(\mathrm{HT})$ in 0.1 $\mathrm{M} \mathrm{HCl}$, and (h) Co-TA spheres after hydrothermal treatment at $100{ }^{\circ} \mathrm{C}$ in $0.1 \mathrm{M}$ $\mathrm{HCl}$. (g) The reaction for the metal-TA coordination in the acid conditions. (i) The possible structure for the Co-TA sphere after HT.

Based on the above results, we speculate the formation mechanism of metal-phenolic coordination spheres mainly includes two steps. In the first step, TA molecules were precrosslinked by formaldehyde. Like other phenols (such as resorcinol), formaldehyde can induce the polymerization of TA, resulting in the formation of TA-formaldehyde resin. In the weak alkaline ethanol/water solution, this polymerization reaction can be well controlled. As a result, low-molecular-weight PTA oligomers were obtained. In the second step, the PTA oligomers were further aggregated together to form metal-phenolic spheres via the metal-ligand crosslinking. Different from the conventional phenols (such as resorcinol), TA has abundant of catechol groups. After crosslinking by formaldehyde, PTA oligomers would still have enough catechol groups to chelate with metal ions to form coordination polymers. When formaldehyde was not used to crosslink the TA, metal-TA coordination rods can be synthesized. This may be due to the fast polymerization rate of metal ions and TA. Comparing with the formation of polymer rods, the formaldehyde crosslinking of polyphenol may weaken the interactions between polyphenol and metal ions by decreasing the numbers of catechol groups. As a result, the metal-ligand assembly can proceed in a mild manner, and the thermodynamic stable spheres are obtained.

Fluorescent nanoprobes have shown promising applications for detection of biomolecules. As a proof-of-concept application, the metal-phenolic coordination spheres were used as effective fluorescence sensing platform for the analysis of nucleic acid 
variants such as miRNA (Figure S9). Fluorophore-labeled single-stranded DNA probe is adsorbed and quenched by the metal-phenolic coordination polymer spheres, whereas fluorescence recovery is observed as the addition of complementary traget miR-21 even down to $2 \mathrm{nM}$ (Figure 4a). This indicates a high affinity of single-stranded nucleic acids rather than double-stranded ones on these spheres. It is well known that double-stranded nucleic acids seal phosphates and nitrogenous bases in the double helix structure. Therefore, we reason that such high affinity is mainly ascribed to the coordination interactions (between metal ions and nitrogenous bases/backbone phosphates) and $\pi-\pi$ stacking interactions (between phenolic rings and nitrogenous base rings). These synergetic interactions could significantly improve the assay performance. The nanomolar sensitivity is consistent with those of traditional DNA probes such as molecular beacons. Morevoer, such nanoprobe system can even distinguish miRNA homogeneous sequences with single nucleotide variation (Figure 4b). This sequence specificity is superior to those of other MOF nanoprobes and traditional DNA probes (Table S2).

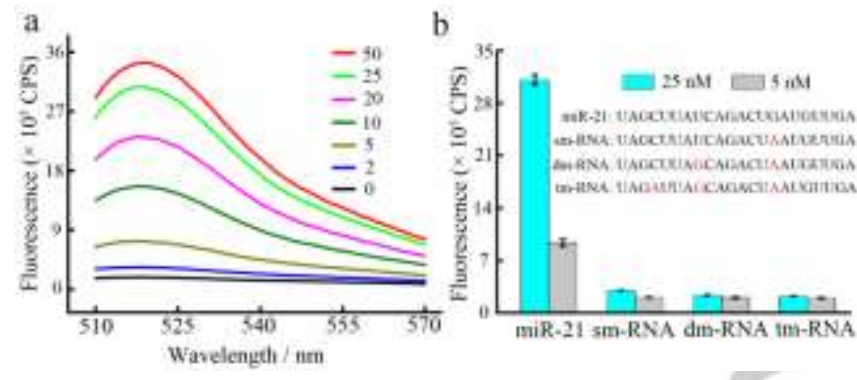

Figure 4. (a) Fluorescent recovery in different concentrations of miR-21 solution, unit: nM. (b) Selectivity for the detection of miR-21.

The conversion of biomass into valuable carbon composites as an efficient non-precious metal catalyst for ORR is desirable and challenging for the development of commercially viable fuelcell technologies. Due to the low cost and nontoxicity of the plant polyphenol and tunable compositions of metal-phenolic coordination polymers, the metal-phenolic coordination spheres were used as a sustainable carbon source to fabricate $M / N-C$ (M $=\mathrm{Fe}, \mathrm{Co}$ ) based catalysts for ORR. Co-TA, Fe-TA and Co(II)$\mathrm{Fe}$ (III)-TA coodination spheres were used as a precursor. Dicyandiamide was used as a $\mathrm{N}$ source. After gridding with coordination polymers and $\mathrm{N}$ source in the mortar, the composites were carbonized at $800{ }^{\circ} \mathrm{C}$ in $\mathrm{N}_{2}$ atmosphere for 3 hours. After the acid etching to remove the exposed metal (or metal carbide) nanoparticles, the carbon catalysts were obtained which were denoted Co-TA-C800, Fe-TA-C800 and Co-Fe-TA$\mathrm{C} 800$ respectively. TEM and $\mathrm{XRD}$ results reveal the successful synthesis of the metal/N-doped carbon composites (Figure S10, $\mathrm{S} 11$ ). The $\mathrm{Co}$ (or $\mathrm{Fe}_{3} \mathrm{C}$ ) nanoparticles are encapsulated by a few layers of graphitic carbon. Element mapping results show that the nitrogen element is doped in the carbon framework uniformly. $\mathrm{N}_{2}$ sorption results show that the BET surface area of Co-TAC800, Fe-TA-C800 and Co-Fe-TA-C800 is $477,328,449 \mathrm{~m}^{2} \mathrm{~g}^{-1}$ respectively (Figure $\mathrm{S} 12$, Table $\mathrm{S} 1$ ). XPS results reveal the existence of nitrogen species in the carbon framework (Figure S13). High-resolution N 1s spectra prove the existence pyridinic and graphitic $\mathrm{N}$ with relative content of 34.3 and 60.1 at\%, which is beneficial to improving the catalytic performance. ${ }^{[12]}$
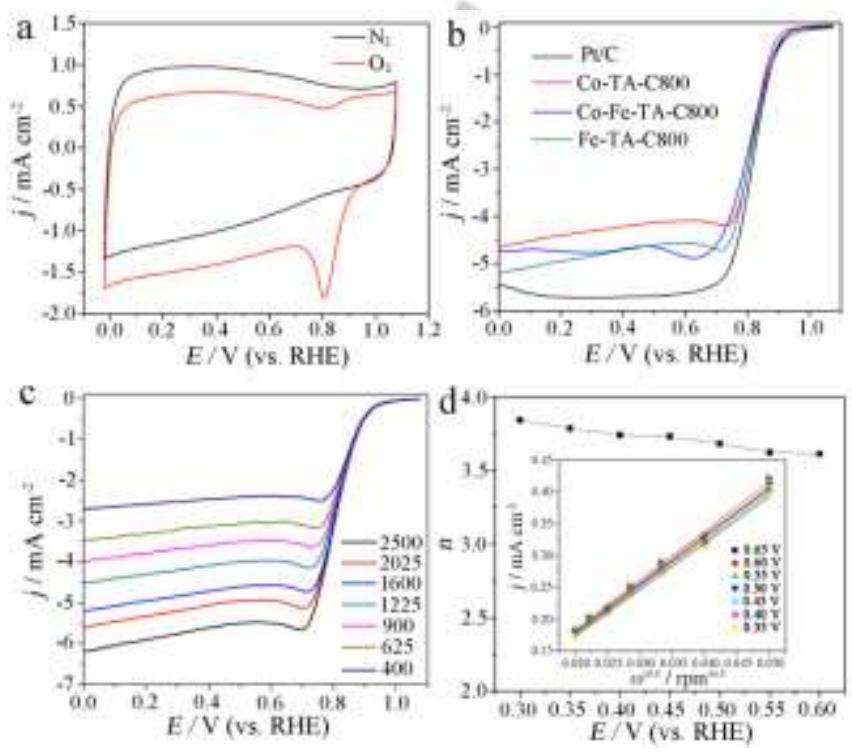

Figure 5. (a) CV curves for Co-Fe-TA-C800. (b) LSV curves for Pt/C, CoTA-C800, Fe-TA-C800 and Co-Fe-TA-C800 in $\mathrm{O}_{2}$-saturated $0.1 \mathrm{M} \mathrm{KOH}$ electrolyte at a rotating speed of $1600 \mathrm{rpm}$. (c) LSV curves for Co-Fe-TA$\mathrm{C} 800$ at different rotating speeds. (d) Electron transfer numbers and K-L plots (inset) for Co-Fe-TA-C800.

The ORR performance of metal-phenolic coordination polymer derived catalysts was evaluated using a rotating disk electrode technique. Cyclic voltammetry (CV) curves reveal a clear oxygen reduction peak for all the materials, implying an oxygen reduction activity (Figure 5a, Figure S14). The linear scan voltammogram (LSV) curves for commercial Pt/C, Co-TA-C800, Fe-TA-C800 and Co-Fe-TA-C800 at the rotating speed of 1600 rpm in $\mathrm{O}_{2}$-saturated $0.1 \mathrm{M} \mathrm{KOH}$ solution show their onset potentials are at $0.95,0.93,0.95$ and $0.96 \mathrm{~V}$ ( $v s$ reversible hydrogen electrode, RHE), respectively (Figure $5 b$ ). The onset potential for Co-Fe-TA-C800 is slightly higher than that of $\mathrm{Pt} / \mathrm{C}$, Co-TA-C800 and Fe-TA-C800, indicating a high catalytic activity. The corresponding limiting current density at $0.30 \mathrm{~V}$ is $5.7,4.3$, 4.8 and $4.8 \mathrm{~mA} \mathrm{~cm}^{-2}$ for Pt/C, Co-TA-C800, Fe-TA-C800 and Co-Fe-TA-C800, respectively. The Koutechy-Levich plots of CoTA-C800, Fe-TA-C800 and Co-Fe-TA-C800 exhibit good linearity with a similar slop (Figure 5c, d, Figure S15, S16), indicating a first-ordered reaction and similar electron transfer numbers $(n)$. The $n$ value at potential of $0.3 \mathrm{~V}$ for Co-TA-C 800 , Fe-TA-C800 and Co-Fe-TA-C800 is $3.91,3.88$ and 3.84 respectively, implying a near four-electron pathway. The catalytic performance is among the best of non-precious metal catalysts reported to date (Table S3). The high catalytic performance may be ascribed to the synergetic catalytic effect between graphitic carbon layers activated by the encapsulated $\mathrm{Fe}_{3} \mathrm{C}$ (or Co) nanoparticles and $\mathrm{Fe}$ (or $\mathrm{Co}$ )/ $/ \mathrm{N}-\mathrm{C}$ species in the catalysts. ${ }^{[10]}$ Moreover, the catalysts derived from metal-phenolic coordination spheres show higher catalytic activity than other metal-phenolic catalysts (Figure S17). Becaused of the enhanced surface area, more numbers of active sites would be exposed. The mass transport would also be enhanced due to the nanoporous framework. Such catalysts also reveal better 
long-term stability than Pt/C (Figure S18). Co-Fe-TA-C800 exhibits higher resistance to the methanol cross-over effect than $\mathrm{Pt} / \mathrm{C}$. These results demonstrate that metal-phenolic coordination polymer spheres are an excellent precursor to fabricate efficient electrode materials.

In summary, a formaldehyde-assisted metal-ligand crosslinking strategy is developed to synthesize metal-phenolic coordination spheres with unique morphology and tailorable compositions. TA molecules were firstly pre-crosslinked by formaldehyde to form PTA oligomers. Then, metal ions were used as a crosslinker to further assemble the PTA oligomers to metal-phenolic coordination spheres. These metal-phenolic coordination spheres can be applied as sensing platforms for the sensitive analysis of nucleic acid variants with single-nucleotide discrimination. It is further demonstrated such colloidal metalphenolic coordination spheres can be used as a precursor to fabricate functional carbon composites. The efficient catalytic activity for oxygen reduction reaction demonstrated that such metal-phenolic coordination spheres were an excellent precursor for the fabrication of carbon based electrode materials. It is believed the formaldehyde-assistant metal-ligand crosslinking strategy would open up new avenues for the synthesis of advanced colloidal spheres with promising applications for biomedicine and electrochemical devices.

\section{Acknowledgements}

J. Wei, G. Wang, and F. Chen contributed equally. This work was financially supported by the National Science Foundation of China (No. 21475102, No. 31671013, No. 21701130 and No. 21705124), the China Postdoctoral Science Foundation (No. 2017M613102), the Fundamental Research Funds for the Central Universities and "Young Talent Support Plan" of Xi'an Jiaotong University.

Keywords: Plant polyphenol• sol-gel chemistry $\bullet$ colloidal sphere coordination polymer $\cdot$ electrocatalysis

[1] [a] M. Oh, C. A. Mirkin, Nature 2005, 438, 651-654; [b] X. M. Sun, Y. D. Li, Angew. Chem. Int. Ed. 2004, 43, 597-601; Angew. Chem. 2004, 116, 607-611; [c] Y. N. Xia, B. Gates, Y. D. Yin, Y. Lu, Adv. Mater. 2000, 12, 693-713; [d] F. Caruso, R. A. Caruso, H. Mohwald, Science 1998, 282, 1111-1114; [e] E. Matijevic, Acc. Chem. Res. 1981, 14, 22-29; [f] J. Liu, N. P. Wickramaratne, S. Z. Qiao, M. Jaroniec, Nat. Mater. 2015, 14, 763-774.

[2] W. Stöber, A. Fink, E. Bohn, J. Colloid. Inter. Sci. 1968, 26, 62-69.

[3] [a] J. Liu, T. Yang, D. W. Wang, G. Q. Lu, D. Zhao, S. Z. Qiao, Nat. Commun. 2013, 4, 2798; [b] W. Li, D. Zhao, Adv. Mater. 2013, 25, 142 149; [c] K. Ai, Y. Liu, C. Ruan, L. Lu, G. Lu, Adv. Mater. 2013, 25, 9981003; [d] J. Liu, S. Z. Qiao, H. Liu, J. Chen, A. Orpe, D. Zhao, G. Q. Lu, Angew. Chem. Int. Ed. 2011, 50, 5947-5951; Angew. Chem. 2011, 123, 6069-6073.

[4] K. Sumida, K. Liang, J. Reboul, I. A. Ibarra, S. Furukawa, P. Falcaro, Chem. Mater. 2017, 29, 2626-2645.
[5] [a] H. Li, M. Eddaoudi, M. O'Keeffe, O. M. Yaghi, Nature 1999, 402, 276-279; [b] S. Kitagawa, R. Kitaura, S. Noro, Angew. Chem. Int. Ed. 2004, 43, 2334-2375; Angew. Chem. 2004, 116, 2388-2430; [c] H. Furukawa, K. E. Cordova, M. O'Keeffe, O. M. Yaghi, Science 2013, 341, 974; [d] J. Liu, L. Chen, H. Cui, J. Zhang, L. Zhang, C. Y. Su, Chem. Soc. Rev. 2014, 43, 6011-6061; [e] S. Qiu, M. Xue, G. Zhu, Chem. Soc. Rev. 2014, 43, 6116-6140; [f] V. Stavila, A. A. Talin, M. D. Allendorf, Chem. Soc. Rev. 2014, 43, 5994-6010; [g] T. Zhang, W. Lin, Chem. Soc. Rev. 2014, 43, 5982-5993; [h] Q.-L. Zhu, Q. Xu, Chem. Soc. Rev. 2014, 43, 5468-5512; [i] A. H. Chughtai, N. Ahmad, H. A. Younus, A Laypkov, F. Verpoort, Chem. Soc. Rev. 2015, 44, 6804-6849; [j] T. Rodenas, I. Luz, G. Prieto, B. Seoane, H. Miro, A. Corma, F. Kapteijn, F. X. Llabres i Xamena, J. Gascon, Nat. Mater. 2015, 14, 48-55; [k] T. R. Cook, Y. R. Zheng, P. J. Stang, Chem. Rev. 2013, 113, 734-777.

[6] [a] X. P. Sun, S. J. Dong, E. K. Wang, J. Am. Chem. Soc. 2005, 127, 13102-13103; [b] Y. M. Jeon, J. Heo, C. A. Mirkin, J. Am. Chem. Soc. 2007, 129, 7480-7481; [c] I. Imaz, D. Maspoch, C. Rodriguez-Blanco, J. M. Perez-Falcon, J. Campo, D. Ruiz-Molina, Angew. Chem. Int. Ed. 2008, 47, 1857-1860; Angew. Chem. 2008, 120, 1883-1886. [d] Y. M. Jeon, G. S. Armatas, J. Heo, M. G. Kanatzidis, C. A. Mirkin, Adv. Mater 2008, 20, 2105-2110; [e] N. R. Champness, Angew. Chem. Int. Ed. 2009, 48, 2274-2275; [f] H. J. Lee, J. We, J. O. Kim, D. Kim, W. Cha, E. Lee, J. Sohn, M. Oh, Angew. Chem. Int. Ed. 2015, 54, 10564-10568; Angew. Chem. 2015, 127, 10710-10714; [g] A. M. Spokoyny, D. Kim, A. Sumrein, C. A. Mirkin, Chem. Soc. Rev., 2009, 38, 1218-1227.

[7] [a] H. Ejima, J. J. Richardson, K. Liang, J. P. Best, M. P. van Koeverden, G. K. Such, J. Cui, F. Caruso, Science 2013, 341, 154-157; [b] J. Guo, Y. Ping, H. Ejima, K. Alt, M. Meissner, J. J. Richardson, Y. Yan, K. Peter, D. von Elverfeldt, C. E. Hagemeyer, F. Caruso, Angew. Chem. Int. Ed. 2014, 53, 5546-5551; Angew. Chem. 2014, 126, 56525657 ; [c] K. Kim, M. Shin, M. Y. Koh, J. H. Ryu, M. S. Lee, S. Hong, H. Lee, Adv. Funct. Mater. 2015, 25, 2402-2410; [d] P. Zhang, H. Li, G. M. Veith, S. Dai, Adv. Mater. 2015, 27, 234-239; [e] J. Guo, B. L. Tardy, A. J. Christofferson, Y. Dai, J. J. Richardson, W. Zhu, M. Hu, Y. Ju, J. Cui, R. R. Dagastine, I. Yarovsky, F. Caruso, Nat. Nano. 2016, 11, 11051111; [f] H. Ejima, J. J. Richardson, F. Caruso, Nano Today 2017, 12 136-148; [g] P. Zhang, L. Wang, S. Yang, J. A. Schott, X. Liu, S. M. Mahurin, C. Huang, Y. Zhang, P. F. Fulvio, M. F. Chisholm, S. Dai, Nat. Commun. 2017, 8, 15020

[8] Q. Wei, R. Haag, Mater. Horiz. 2015, 2, 567-577.

[9] [a] K. Ai, Y. Liu, C. Ruan, L. Lu, G. Lu, Adv. Mater. 2013, 25, 998-1003; [b] Z. Wang, Y. Xie, Y. Li, Y. Huang, L. R. Parent, T. Ditri, N. Zang, J. D. Rinehart, N. C. Gianneschi, Chem. Mater. 2017, 29, 8195-8201; [c] M. Xiao, Y. Li, M. C. Allen, D. D. Deheyn, X. Yue, J. Zhao, N. C. Gianneschi, M. D. Shawkey, A. Dhinojwala, ACS Nano, 2015, 9, 5454 5460; [d] M. Xiao, Z. Hu, Z. Wang, Y. Li, A. D. Tormo, N. L. Thomas, B. Wang, N. C. Gianneschi, M. D. Shawkey, A. Dhinojwala, Sci. Adv. 2017, 3, e1701151.

[10] [a] J. Wei, Y. Liang, Y. Hu, B. Kong, J. Zhang, Q. Gu, Y. Tong, X. Wang, S. P. Jiang, H. Wang, Angew. Chem. Int. Ed. 2016, 55, 12470-12474; Angew. Chem. 2016, 128, 12658-12662; [b] J. Wei, Y. Liang, Y. Hu, B. Kong, G. P. Simon, J. Zhang, S. P. Jiang, H. Wang, Angew. Chem. Int. Ed. 2016, 55, 1355-1359; Angew. Chem. 2016, 128, 1377-1381.

[11] X. Wang, L. L. Lu, Z. L. Yu, X. W. Xu, Y. R. Zheng, S. H. Yu, Angew. Chem. Int. Ed. 2015, 54, 2397-2401; Angew. Chem. 2015, 127, 2427 2431.

[12] [a] K. Gong, F. Du, Z. Xia, M. Durstock, L. Dai, Science 2009, 323, 760764; [b] D. Guo, R. Shibuya, C. Akiba, S. Saji, T. Kondo, J. Nakamura, Science 2016, 351, 361-365. 
Entry for the Table of Contents (Please choose one layout)

Layout 2:

\section{COMMUNICATION}

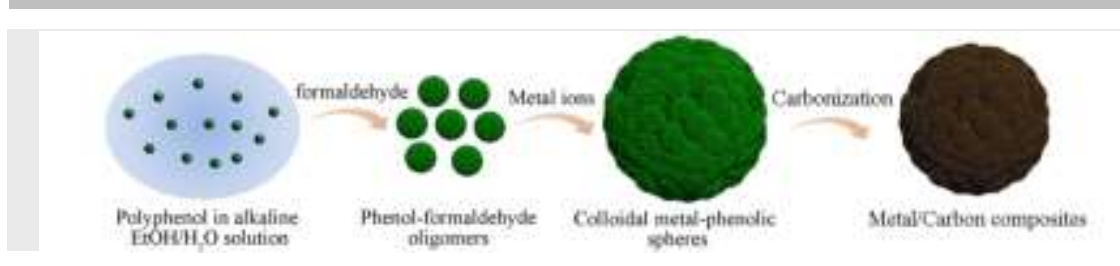

A formaldehyde-assisted metal-ligand crosslinking strategy is developed for the synthesis of metal-phenolic coordination spheres with uniform diameter and tunable compositions. The coordination spheres can be used as sensing platforms for the analysis of nucleic acid variants with single-nucleotide discrimination, and a versatile precursor for electrode materials with high electrocatalytic performance.
J. Wei, G. Wang, F. Chen, M. Bai, Y. Liang, H. Wang, D. Zhao, Y. Zhao*

\section{Page No. - Page No.}

Sol-gel Synthesis of Metal-Phenolic Coordination Spheres and Their Derived Carbon Composites 


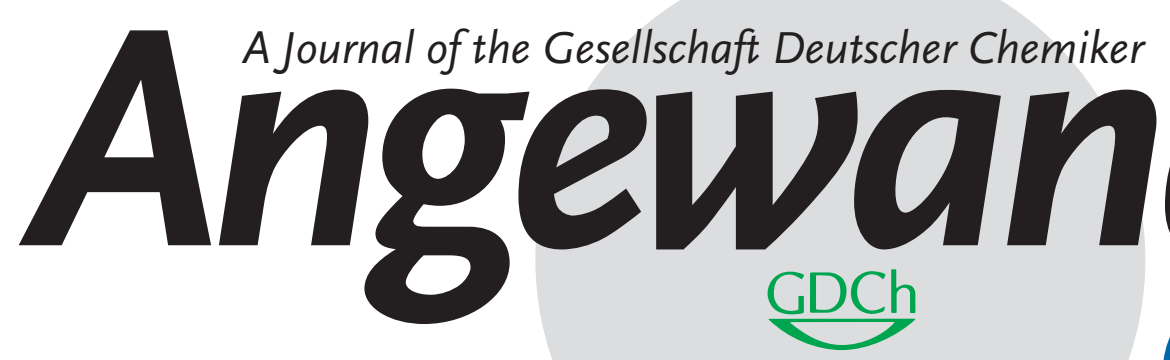

International Edition

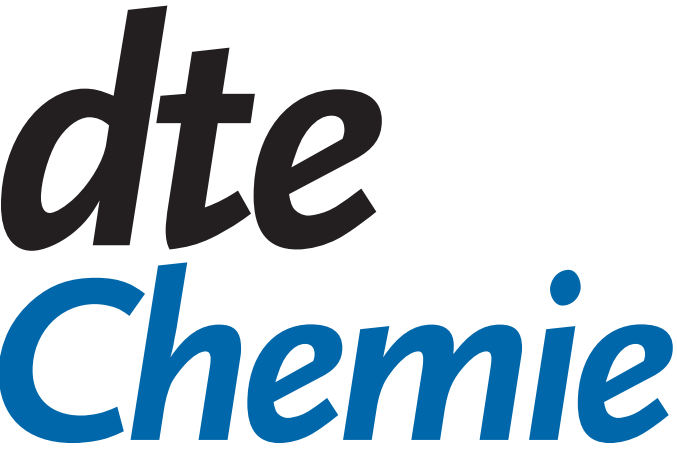

www.angewandte.org

\section{Accepted Article}

Title: Sol-gel Synthesis of Metal-Phenolic Coordination Spheres and Their Derived Carbon Composites

Authors: Jing Wei, Gen Wang, Feng Chen, Min Bai, Yan Liang, Huanting Wang, Dongyuan Zhao, and Yongxi Zhao

This manuscript has been accepted after peer review and appears as an Accepted Article online prior to editing, proofing, and formal publication of the final Version of Record (VoR). This work is currently citable by using the Digital Object Identifier (DOI) given below. The VoR will be published online in Early View as soon as possible and may be different to this Accepted Article as a result of editing. Readers should obtain the VoR from the journal website shown below when it is published to ensure accuracy of information. The authors are responsible for the content of this Accepted Article.

To be cited as: Angew. Chem. Int. Ed. 10.1002/anie.201805781

Angew. Chem. 10.1002/ange.201805781

Link to VoR: http://dx.doi.org/10.1002/anie.201805781

http://dx.doi.org/10.1002/ange.201805781 